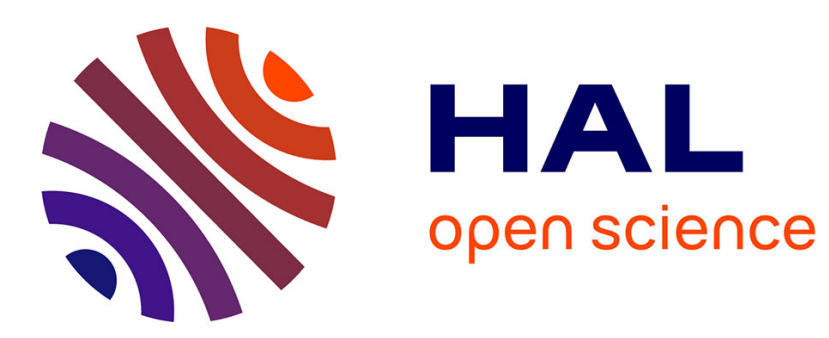

\title{
Phase Noise Model Selection for Sub-THz Communications
}

\author{
Simon Bicaïs, Jean-Baptiste Doré
}

\section{To cite this version:}

Simon Bicaïs, Jean-Baptiste Doré. Phase Noise Model Selection for Sub-THz Communications. IEEE Global Communications Conference 2019, Dec 2019, Waikoloa, United States. hal-02265725

\section{HAL Id: hal-02265725 \\ https://hal.science/hal-02265725}

Submitted on 12 Aug 2019

HAL is a multi-disciplinary open access archive for the deposit and dissemination of scientific research documents, whether they are published or not. The documents may come from teaching and research institutions in France or abroad, or from public or private research centers.
L'archive ouverte pluridisciplinaire HAL, est destinée au dépôt et à la diffusion de documents scientifiques de niveau recherche, publiés ou non, émanant des établissements d'enseignement et de recherche français ou étrangers, des laboratoires publics ou privés. 


\title{
Phase Noise Model Selection for Sub-THz Communications
}

\author{
Simon Bicaïs Jean-Baptiste Doré \\ CEA-Leti, Minatec Campus, Grenoble, France \\ simon.bicais@cea.fr
}

\begin{abstract}
To face the exponential data traffic growth, the sub-THz spectrum $(100-300 \mathbf{G H z})$ is envisioned for wireless communications. However, sub-THz systems are critically impacted by the strong phase noise of high frequency oscillators. This paper discusses the appropriate choice of phase noise model for sub-THz communications. Two phase noise models are introduced and compared: one correlated, accurate but complex, and another uncorrelated, analytically simpler. The expression of the likelihood ratio enables us to propose an analytical condition to select the best of the two models for a measured oscillator spectral characteristic. Numerical simulations are performed with realistic phase noise generated according to a state-of-the-art sub-THz oscillator and show that an uncorrelated Gaussian process is appropriate to model the impact of phase noise in sub-THz systems. Eventually, the proposed results are applied to link adaptation in the presence of phase noise in order to choose the most robust scheme between a coherent and a differential modulation.

Index Terms-Sub-THz communications, Oscillator, Phase
\end{abstract} noise, Wiener Phase Noise, Gaussian distribution.

\section{INTRODUCTION}

With a specific demand for wireless connectivity, the current exponential data traffic growth will lead to a $100+$ Gbit/s requirement within few years. To meet this demand, the large bands available in the $s u b-T H z$ spectrum $(100-300 \mathrm{GHz})$ are studied for wireless communications [1]. To reach $100+$ Gbit/s data rates, classical coherent architectures are combined with high spectral efficiency schemes. This entails numerous constraints on the design of radio-frequency components especially at the oscillator level. Indeed, high frequency oscillators severely impair sub-THz systems with Phase Noise (PN) [2]. It is hence essential to discuss the PN model selection to design dedicated signal processing and thus performant schemes.

A wide range of $\mathrm{PN}$ models have been introduced in the literature: Non-linear [3], Tikhonov [4], Wiener [5], ... The selection of the PN model is application dependent. For millimeter-wave systems, the PN is usually modeled by the superposition of a correlated contribution (colored PN) and an uncorrelated one (white PN floor) [6]. However, sub-THz systems exploit wider bands and thus require to reconsider the PN model. It has been shown in [5] that performance of wide-band systems are significantly more impacted by the uncorrelated contribution of PN than the correlated one. This result prompts us to compare the efficiency of an uncorrelated Gaussian PN model to the common correlated one used for millimeter-wave, superposition of Wiener PN and Gaussian PN.

In this work, we intend to provide analytical tools for sub$\mathrm{THz}$ communication design and circuit specifications. Firstly, we present two PN models: one correlated (Wiener PN + Gaussian PN) and another uncorrelated (Gaussian PN). Secondly, these two models are confronted by means of numerical simulations as well as an analytical analysis. The accuracy of

The research leading to these results received funding from the French National Research Agency (ANR-17-CE25-0013) within project BRAVE. the uncorrelated Gaussian PN model is first checked with a Pearson's $\chi^{2}$ goodness of fit test. We next derive the closedform expression of the likelihood-ratio of the two models. Thereupon, an analytical condition is proposed to determine whether the correlated or the uncorrelated model is the most efficient given a system bandwidth and an oscillator spectral characterization. Numerical simulations are performed with realistic PN generated according to a state-of-the-art sub- $\mathrm{THz}$ oscillator. Simulation results show that an uncorrelated Gaussian process is appropriate to model the impact of PN in sub$\mathrm{THz}$ systems. Thirdly, the proposed results on model selection are applied to link adaptation in the presence of PN. Upon the analysis of the Bit-Error-Rate (BER) of phase modulated signals, we are able to choose the most robust scheme between a coherent and a differential modulation.

The remainder of this paper is structured as follows. Section II describes the considered communication system and oscillator. Section III presents the correlated and uncorrelated PN models. Section IV is dedicated to the comparison of these two models in order to select the most efficient one for a given system. Section $\mathrm{V}$ applies the results on model selection to link adaptation while Section VI eventually draws some conclusions.

\section{SySTEM DESCRIPTION}

\section{A. Oscillator}

The modeling of the complex output $v$ of a practical oscillator with central frequency $f_{0}$ is given by

$$
v(t)=(1+\varepsilon(t)) \exp \left(j 2 \pi\left(f_{0}+\zeta\right) t+j \phi(t)\right),
$$

where $\varepsilon, \zeta$ and $\phi$ denote respectively the amplitude noise, the Carrier Frequency Offset (CFO) and the PN. The amplitude noise $\varepsilon$ is mitigated by applying a limiter on the oscillator output and is considered negligible [7]. It is also assumed that the receiver is synchronized in frequency s.t. the CFO term $\zeta$ is compensated. When it comes to $\mathrm{PN}$, the common figure to characterize the performance of an oscillator is its SingleSide-Band (SSB) spectrum $\mathcal{L}(f)$. It is obtained throughout the following measurements

$$
\mathcal{L}(f)=\frac{S_{v}\left(f_{0}+f\right)}{P_{v}},
$$

with $S_{v}$ the Power Spectral Density (PSD) and $P_{v}$ the power of the oscillator output $v$. Though the total power of an oscillator should ideally be concentrated in $f_{0}$ and the PSD of the oscillator a pure tone, PN induces the power to be spread over frequencies around $f_{0}$. We denote $S_{\phi}$ the PSD of PN $\phi$. For large $f$, the small angle approximation $e^{j \phi} \simeq 1+j \phi$ leads to $S_{\phi}(f) \simeq \mathcal{L}(f)$. The latter equation bonds the $\mathrm{PN} \phi$ and the oscillator output $v$. This link enables us to calibrate the PN model on the oscillator spectral characterizations. 


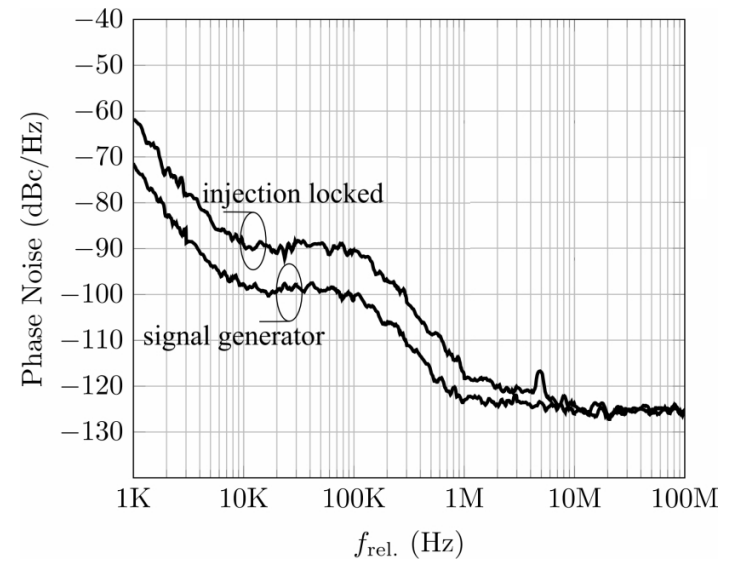

Fig. 1: PSD of the considered $200 \mathrm{GHz}$ oscillator [8].

In this work, we intend to confront PN models on realistic observations. We therefore select a state-of-the-art $200 \mathrm{GHz}$ oscillator [8], whose PSD is illustrated in Fig. 1. The simulated PN will correspond to this oscillator by respecting the spectral characteristics measured in Fig. 1. We refer the reader to [5] for PN simulation based on oscillator spectral characterizations and also to [9] for simulation of power law noise.

\section{B. Channel}

The recent measurement campaigns [10] have confirmed the expectations from theory that the line-of-sight component prevails in sub-THz channels, hence considered as frequency flat. We study a single carrier communication system ${ }^{1}$ impacted by oscillator PN. It is assumed that the channel is ideally equalized ${ }^{2}$ and the receiver synchronized in time and frequency. Subsequently, we consider the discrete-time symbol model of an Additive White Gaussian Noise (AWGN) channel impacted by $\mathrm{PN}$. The received symbol at instant $k$ is expressed by

$$
r_{k}=s_{k} e^{j \phi_{k}}+w_{k},
$$

where $s_{k}$ is the modulated symbol with duration $T$ and $w_{k} \sim \mathbb{C} \mathcal{N}\left(0, \sigma_{w}^{2}=N_{0} / T\right)$. The spectral density of the white noise is denoted $N_{0}$. The discrete stochastic process $\phi_{k}$ represents the oscillator PN to be modeled. Further, the discretetime symbol model in Eq. (3) is discussed in [11]. Conditions on the PN intensity and the sampling rate of the system are given for this model to be accurate. It is also worth mentioning that communication systems may use separate oscillators at the transmitter and the receiver. In this case, both PN contributions must be added together.

\section{Phase NoISE Models}

We present in this section two PN models. The first one is a correlated model based on the physical modeling of the PN generation in oscillators. This model is frequently used for millimeter-wave systems [6] and describes the superposition of a correlated contribution (colored PN) and an uncorrelated one (white PN floor). The second one is an uncorrelated model describing only the white PN floor. Its mathematical expression is hence simple and commonly exploited to optimize the signal processing for PN channels [12].

\footnotetext{
${ }^{1}$ Though the presented results may also be exploited for channel bounding or multi-carrier modulation schemes.

${ }^{2}$ The phase shift of the channel is compensated and the propagation gain is normalized.
}

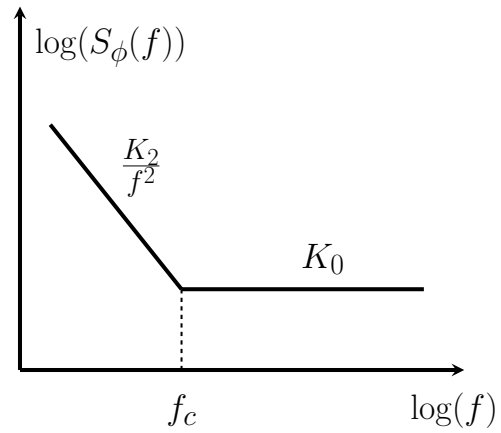

Fig. 2: PSD of the considered correlated PN model.

\section{A. Correlated phase noise}

PN generation in oscillators is due to the transformation of amplitude fluctuations into phase fluctuations. In practical systems, free-running oscillators are stabilized by means of a Phase-Locked Loop (PLL). With respect to the linear timeinvariant model of the PLL, the PN generation mechanism may be modeled as an amplification and an integration of the noise sources present in the circuits [5] [7]. The integration of noise sources by the PLL gives a cumulative and correlated nature to PN. We make the assumption that the circuitry is only subject to white noise ${ }^{3}$. Accordingly, the oscillator PN $\phi_{k}$ may be modeled by the superposition of a Wiener (Gaussian random-walk) process $\phi_{w, k}$ and a Gaussian one $\phi_{g, k}$ [7] i.e.

$$
\phi_{k}=\phi_{w, k}+\phi_{g, k} \text {. }
$$

The Gaussian PN $\phi_{g, k} \sim \mathcal{N}\left(0, \sigma_{g}^{2}\right)$ is caused by the amplification of the thermal noise in the oscillator. The Wiener PN is defined by

$$
\phi_{w, k}=\phi_{w, k-1}+\delta \phi_{w, k},
$$

where $\delta \phi_{w, k} \sim \mathcal{N}\left(0, \sigma_{w}^{2}\right)$. It expresses the integration by the PLL and hence the cumulative nature of PN. Fig. 2 presents the PSD described by this PN composed of two characteristics. The uncorrelated Gaussian process with variance $\sigma_{g}^{2}=K_{0} / T$ has a flat spectrum of density $K_{0}$. The correlated Wiener PN presents a Lorentzian characteristic (i.e. $1 / f^{2}$ ) [7] with density $K_{2}$. The variance of the Wiener increment verifies $\sigma_{w}^{2}=4 \pi^{2} K_{2} T$ [7]. The variances $\sigma_{g}^{2}$ and $\sigma_{w}^{2}$ of the two processes are bonded by the corner frequency $f_{c}^{2}=K_{2} / K_{0}$. We use hereafter the normalized corner frequency $f_{c} \cdot T$ expressed as a fraction of the band. In the following, we will refer to this model as $M_{W}$ for short. Based on the physical modeling of the PN generation in oscillators, this model is frequently used in the literature for its accuracy - see [5] and references therein. However its complexity motivates the introduction of an uncorrelated Gaussian PN model.

\section{B. Uncorrelated phase noise}

By definition, modeling a stochastic process represents a trade-off between the accuracy and the complexity of description. Since the development of communication systems highly benefits from analytical analyses, the low analytical complexity of a model can be preferred over its accuracy. By means of illustration, the Gaussian PN model has already been exploited in [12] to optimize constellations for PN

\footnotetext{
${ }^{3}$ The influence of flicker noise is disregarded in this paper
} 
TABLE I: Spectral density $K_{0}(\mathrm{dBc} / \mathrm{Hz})$ of the white PN floor.

\begin{tabular}{|cc|c|c|c|}
\cline { 3 - 5 } \multicolumn{1}{c|}{} & \multicolumn{3}{c|}{ Bandwidth $1 / T$} \\
\cline { 3 - 5 } \multicolumn{1}{c|}{} & & $100 \mathrm{MHz}$ & $1 \mathrm{GHz}$ & $10 \mathrm{GHz}$ \\
\hline Strong PN & $\sigma_{g}^{2}=10^{-1}$ & -90 & -100 & -110 \\
\hline Medium PN & $\sigma_{g}^{2}=10^{-2}$ & -100 & -110 & -120 \\
\hline Low PN & $\sigma_{g}^{2}=10^{-3}$ & -110 & -120 & -130 \\
\hline
\end{tabular}

channels and also in [13] to improve demodulation in the presence of PN. We thus turn away from the accurate but complex $M_{W}$ to consider the Gaussian PN model. Besides its mathematical convenience, the Gaussian distribution is also a relevant PN model for wide-band systems. The work in [5] has studied separately the influences of the different PN spectrum regions on communications. It has exhibited a strong dependence with the system bandwidth $1 / T$. In particular, when considering high symbol rate systems, the oscillator noise floor - characteristic $K_{0}$ in Fig. 2 - represents the greatest contribution to the overall PN. This result may also be appreciated by evaluating the ratio

$$
\gamma=\frac{\sigma_{w}^{2}}{\sigma_{g}^{2}}=4 \pi^{2} f_{c}^{2} T^{2} .
$$

For wide-band systems, $f_{c} \ll 1 / T$ leads to $\sigma_{w}^{2} \ll \sigma_{g}^{2}$. It follows that the Wiener PN becomes negligible compared to the Gaussian PN. For these reasons, it is worth considering a Gaussian PN model denoted $M_{G}$. The oscillator PN $\phi_{k}$ is described by a truncated Gaussian distribution,

$$
\phi_{k} \sim \mathcal{N}\left(0, \sigma_{g}^{2}\right) .
$$

As the phase is naturally bounded by $(-\pi, \pi]$, the Gaussian distribution must be truncated. The probability density function is normalized ${ }^{4}$ by $\lambda=\left(F_{\mathcal{N}}(\pi)-F_{\mathcal{N}}(-\pi)\right)^{-1}$ to ensure that $\int_{-\pi}^{\pi} p(\phi) d \phi=1$, where $F$ denotes the cumulative distribution function. Returning to oscillator characterization, Table I presents some values of spectral density $K_{0}$ of the white PN floor for different variances $\sigma_{g}^{2}$ and system bandwidth $1 / T$. The following work evaluates the efficiency of $M_{G}$ to model PN observations. It is clear from Eq. (6) that the accuracy of $M_{G}$ is function of the normalized corner frequency $f_{c} \cdot T$.

\section{Model Selection}

We have previously introduced two PN models: $M_{W}$ correlated with a complex and accurate description of PN, and $M_{G}$ uncorrelated with a simple mathematical expression. In this section, we first evaluate the accuracy of $M_{G}$, the Gaussian distribution, to model PN observations. Then, we compare the likelihood of the correlated $M_{W}$ and uncorrelated $M_{G}$ models in order to select the most efficient one given the system bandwidth and the oscillator spectral characterization.

\section{A. Accuracy of the Gaussian model}

To assess the accuracy of $M_{G}$, we perform a Pearson's $\chi^{2}$ goodness of fit test on simulated PN. By doing so, we intend to determine the systems parameters, communication bandwidth and oscillator spectral characteristics, for which

\footnotetext{
${ }^{4}$ It is worth mentioning that most of algorithms are not subject to a normalization of the likelihood function and hence a factor multiplication does not increase the complexity.
}

$M_{G}$ models accurately the PN observations. The simulated PN samples are generated either according to $M_{W}$, considered as an accurate and realistic PN model, or to the oscillator described in Fig. 1. First, the goodness of fit test is described, and then, the simulations results are presented.

1) Pearson's $\chi^{2}$ goodness of fit test: The aim of this subsection is to establish whether an observed PN frequency distribution differs from a truncated Gaussian distribution. In other words, we operate normality tests on simulated PN. Let $\phi=\left\{\phi_{k}\right\}_{1<k<N}$ denote the observed values of random PN and $N$ the number of symbols within a communication frame. Two different communication frames hence represent two independent realizations of PN. The null hypothesis can now be described as

$$
H_{0}: M_{G} \text { is consistent with the observations } \phi .
$$

To test this hypothesis, a Neyman-Pearson approach is a reasonable choice. This approach is designed for binary detection problem with a given significance $\alpha$ [14]. The hypothesis testing $\delta$ takes the following form

$$
\delta(\phi)= \begin{cases}\text { accept } H_{0}, & \text { if } S(\phi)<\lambda_{c}, \\ \text { reject } H_{0}, & \text { otherwise, }\end{cases}
$$

where $S$ is the test statistic of observed samples $\phi$ compared to a decision threshold $\lambda_{c}$ which maximizes the probability of detection. Further, we exploit the common Pearson's $\chi^{2}$ goodness of fit to test the observations against the null hypothesis $H_{0}$. In this case, the value of the statistic $S$ is

$$
S(\phi)=N \sum_{i=1}^{M} \frac{\left(\frac{O_{i}(\phi)}{N}-p_{i}\right)^{2}}{p_{i}},
$$

where $O_{i}$ is the number of observations $\phi_{k} \in I_{i}$, while $M$ denotes the number of intervals $I_{i}$ partitioning $(-\pi, \pi]$ and $p_{i}=\operatorname{Pr}\left(\phi_{k} \in I_{i}\right)$. To determine the goodness of fit we compare the value of $S(\phi)$ to a $\chi_{M-2}^{2}$ distribution with $M-2$ degrees of freedom ${ }^{5}$. The critical value is given by $\lambda_{c}=F_{\chi_{M-2}^{2}}^{-1}(1-\alpha)$, where $\alpha$ is the given significance.

2) Simulations results: We first perform the $\chi^{2}$ test on PN samples generated according to $M_{W}$, with significance $\alpha=0.1$. Fig. 3 illustrates the frequency of accepting $H_{0}$ as a function of the normalized corner frequency $f_{c} \cdot T$. This confirms the aforementioned intuition in Eq. (6) that $M_{G}$ is accurate when $f_{c} \ll 1 / T$. The value 0.9 of the frequency of accepting is consistent with the significance of 0.1 . It must be pointed out that $N$, the length of the studied sequence, has a great influence on the selection of the model. For long sequences, the impact of the PN correlation is significant and cannot be neglected. Secondly, we test $H_{0}$ against realistic PN samples simulated according to the oscillator described in Fig. 1. The frequency of choosing $\delta(\phi)=H_{0}$ is presented in Fig. 4 as a function of the system bandwidth $1 / T$. Properties observed in Fig. 4 and the resulting conclusions are similar to the ones of Fig. 3. We have confirmed with the Pearson's $\chi^{2}$ goodness of fit test that the uncorrelated Gaussian PN model $M_{G}$ is accurate if the corner frequency remains small compared to the system bandwidth. However, we have not yet

\footnotetext{
${ }^{5}$ The variance is estimated from observations, the mean is not
} 


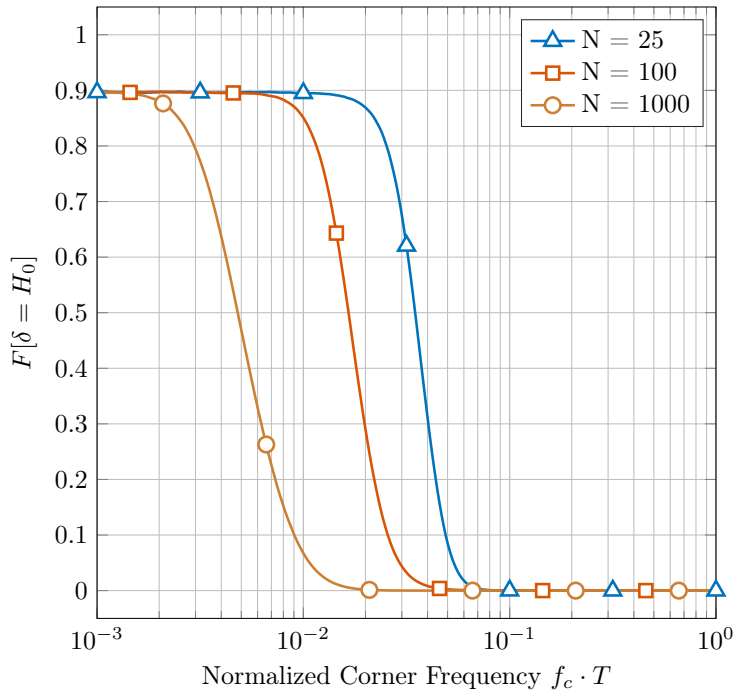

Fig. 3: Frequency of accepting the Gaussian model $H_{0}$ with a $\chi^{2}$ goodness of fit test for simulated correlated PN.

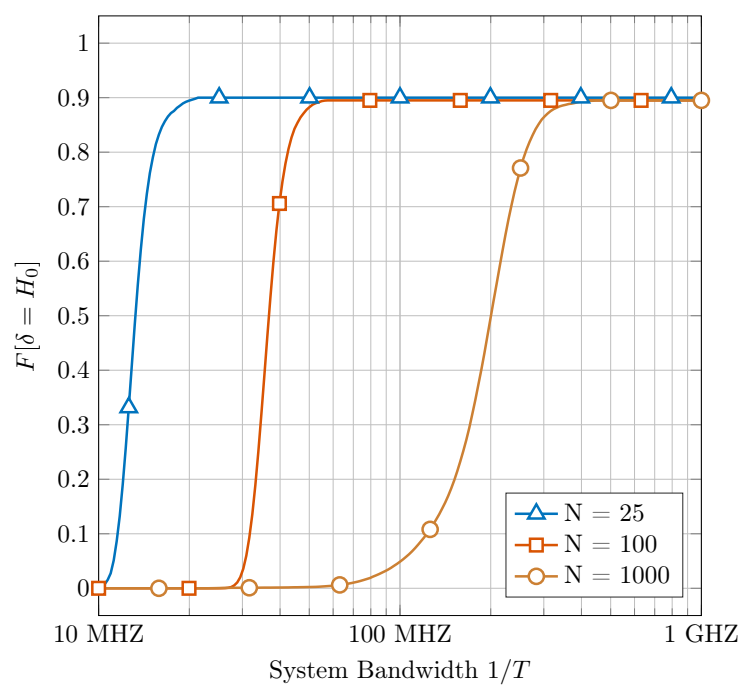

Fig. 4: Frequency of accepting the Gaussian model $H_{0}$ with a $\chi^{2}$ goodness of fit test for simulated realistic PN.

derived an analytical expression of the dependence between the accuracy of $M_{G}$ and the system features $N$ and $f_{c} \cdot T$. This is carried out in the following subsection.

\section{B. Models comparison}

We have previously witnessed the limitations of the Neyman-Pearson approach. The properties of $M_{G}$ have been highlighted, still they have not been expressed analytically. To do so, we compare the efficiency of $M_{W}$ and $M_{G}$ to model some PN observations $\phi=\left\{\phi_{k}\right\}_{1 \leq k \leq N}$. We recall that $N$ denotes the number of symbols within a frame and that different frames represent independent realizations of PN.

1) Log-likelihood ratio: Given the oscillator PSD, the comparison between $M_{G}$ and $M_{W}$ can be achieved by evaluating the Log-Likelihood Ratio (LLR) of the models for observations $\phi$, i.e.

$$
\Lambda_{G / W}=\ln \left(\frac{P\left(\phi \mid M_{G}\right)}{P\left(\phi \mid M_{W}\right)}\right)
$$

denoted $\Lambda$ for brevity. While the sign of $\Lambda$ infers the model to select $\left(M_{G}\right.$ if $\Lambda \geq 0, M_{W}$ otherwise), its absolute value quantifies the reliability of this decision. So, $|\Lambda|$ small leads to the conclusion that $M_{G}$ and $M_{W}$ are equally likely. Conversely, a high value of $|\Lambda|$ indicates that the observations are particularly in favor of one model over the other. To derive the expression of $\Lambda$, we must express the joint distributions $p\left(\phi \mid M_{G}\right)$ and $p\left(\phi \mid M_{W}\right)$. Regarding $M_{G}, \phi_{k}$ are i.i.d.. It is hence straightforward from Eq. (7) that

$$
p\left(\phi \mid M_{G}\right)=\left(2 \pi \sigma_{g}^{2}\right)^{-\frac{N}{2}} \cdot \exp \left(-\sum_{k=1}^{N} \frac{\phi_{k}^{2}}{2 \sigma_{g}^{2}}\right) .
$$

As for $M_{W}, \mathrm{PN}$ is correlated and we must start by applying the conditional chain rule. Denoting $\phi_{k-1}=\left(\phi_{1}, \cdots, \phi_{k-1}\right)$,

$$
p\left(\phi \mid M_{W}\right)=\prod_{k=1}^{N} p\left(\phi_{k} \mid M_{W}, \phi_{k-1}\right)=\prod_{k=1}^{N} p\left(\phi_{k} \mid M_{W}, \phi_{k-1}\right) \text {. }
$$

The latter equality holds as $M_{W}$ is a Markov chain of order 1 . To evaluate the factors in the previous equation, we next define $\Delta \phi_{k}=\phi_{k}-\phi_{k-1}$ which yields

$$
\begin{array}{r}
p\left(\phi_{k} \mid M_{W}, \phi_{k-1}\right) \sim \mathcal{N}\left(\phi_{k-1}, \sigma_{g}^{2}(2+\gamma)\right), \\
=p\left(\Delta \phi_{k} \mid M_{W}\right) \sim \mathcal{N}\left(0, \sigma_{g}^{2}(2+\gamma)\right),
\end{array}
$$

where $\gamma=\sigma_{w}^{2} / \sigma_{g}^{2}$ as defined in Eq. (6). Substituting Eq. (14) into (13), we have

$$
p\left(\phi \mid M_{W}\right)=\left(2 \pi \sigma_{g}^{2}(2+\gamma)\right)^{-\frac{N}{2}} \cdot \exp \left(-\sum_{k=1}^{N} \frac{\Delta \phi_{k}^{2}}{2 \sigma_{g}^{2}(2+\gamma)}\right) .
$$

At last, we obtain the closed-form expression of the LLR:

$$
\Lambda=\frac{N}{2} \ln (2+\gamma)+\sum_{k=1}^{N} \frac{\Delta \phi_{k}^{2}}{2 \sigma_{g}^{2}(2+\gamma)}-\sum_{k=1}^{N} \frac{\phi_{k}^{2}}{2 \sigma_{g}^{2}}
$$

which completes our derivation.

2) Simulation results with correlated phase noise: Let us now consider that the PN observations are generated by a $M_{W}$ process and study the expected value $E[\Lambda]$. Given the system features $N$ and $f_{c} \cdot T$, the expected value of the LLR yields the selected model in average. Since $M_{W}$ is considered as an accurate and realistic PN model, studying the expected value of the LLR enables us to characterize analytically the observed properties in Fig. 3 and 4. When $\phi_{k}$ are generated according to $M_{W}$, we have in Eq. (16)

$$
\sum_{k=1}^{N} \frac{\Delta \phi_{k}^{2}}{\sigma_{g}^{2}(2+\gamma)} \sim \chi_{N}^{2}, \text { with } E\left[\chi_{N}^{2}\right]=N
$$

Then, the second sum in Eq. (16) has for expected value

$$
\begin{aligned}
E\left[\sum_{k=1}^{N} \phi_{k}^{2}\right] & =\sum_{k=1}^{N} E\left[\left(\phi_{w, k}+\phi_{g, k}\right)^{2}\right], \\
& =\sum_{k=1}^{N} E\left[\phi_{w, k}^{2}\right]+E\left[2 \phi_{w, k} \cdot \phi_{g, k}\right]+E\left[\phi_{g, k}^{2}\right], \\
& =\sigma_{g}^{2} \cdot \sum_{k=1}^{N}(k \cdot \gamma+1)=\sigma_{g}^{2}\left(\frac{N(N+1)}{2} \gamma+N\right) .
\end{aligned}
$$




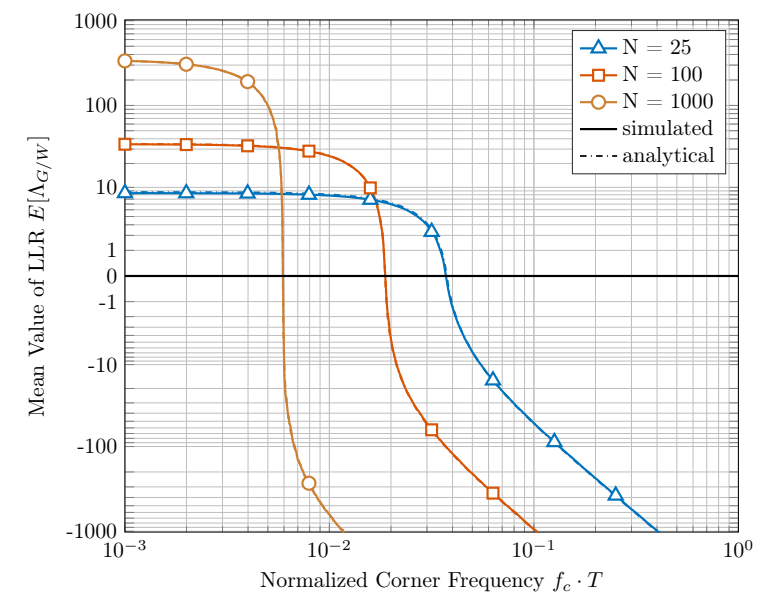

Fig. 5: Mean value of LLR $\Lambda_{G / W}$ on simulated correlated PN.

It follows that the expected value of the LLR is given by

$$
E[\Lambda]=\frac{N}{2}\left(\ln (2+\gamma)-\gamma \cdot \frac{N+1}{2}\right) .
$$

Several remarks must be mentioned with regard to this expression. Firstly, $E[\Lambda] \propto-\gamma \cdot N$ confirms the simulation results that the longer the sequence, the lower the normalized corner frequency must be to ensure that $M_{G}$ remains an accurate model. Furthermore, we are now able to give a condition on $N$ and $f_{c} \cdot T$ for the correlated PN to be negligible. By evaluating the inequality $E[\Lambda] \geq 0$ with a 1st-order approximation, we claim that the PN is appropriately modeled by $M_{G}$ if

$$
N \cdot f_{c}^{2} \cdot T^{2} \leq \frac{\ln (2)}{2 \pi^{2}} .
$$

Regarding communication design, the condition in Eq. (20) may either be exploited to specify the normalized corner frequency $f_{c} \cdot T$ or to set the length $N$ of the frame. Secondly, when the PN is generated from $M_{W}$ with $\gamma$ small, the value of $\Lambda$ may be greater than zero. The LLR actually penalizes the complexity of $M_{W}$ in comparison to $M_{G}$. This illustrates the Occam's razor: if both $M_{G}$ and $M_{W}$ accurately model the observations, then the simplest model should be favored. Eventually, Fig. 5 presents the mean LLR value for different frame lengths with $M_{W}$ simulated PN. Fig. 5 and condition in Eq. (20) may be exploited to select the best PN model $-M_{G}$ if $E[\Lambda] \geq 0, M_{W}$ otherwise - with regard to system features $N$ and $f_{c} \cdot T$.

3) Simulation results with realistic $P N$ : In this paragraph, the simulation results are presented for a realistic PN corresponding to the sub- $\mathrm{THz}$ oscillator described in the Fig. 1. The Fig. 6 shows the mean value of the LLR as a function of the system bandwidth $1 / T$ for several frame lengths $N$. The analytical curves correspond to the expected value of the LLR if the PN samples where generated according to $M_{W}$ with characteristics $K_{0}$ and $K_{2}$ measured on Fig. 1. We can deduce from these results that the condition given in Eq. (20) does indeed ensure that an uncorrelated PN model is valid. Nevertheless, it can be observed that this analytical condition is slightly conservative in view of the results obtained by simulation. This may be explained by the fact that the correlated PN of the oscillator close to the center frequency is not as strong

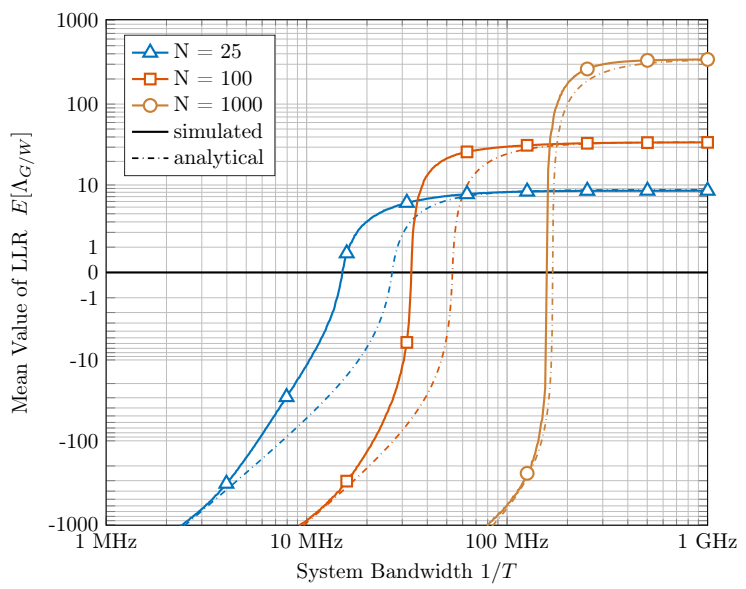

Fig. 6: Mean value of LLR $\Lambda_{G / W}$ on simulated realistic PN.

as the one described by a Lorentzian characteristic. In other words, $M_{W}$ models a stronger correlated PN contribution than the one measured in practice. Furthermore, considering that the bandwidths of sub-THz systems will be of the order of the $\mathrm{GHz}$ [15], we can conclude from these results that an uncorrelated Gaussian model is appropriate to describe the $\mathrm{PN}$ in sub-THz communications.

\section{Application to Link Adaptation}

The objective of this section is to give the reader some application examples. In particular, it aims to illustrate that the proposed model selection may be exploited to adapt the modulation with regards to the oscillator PN performance.

\section{A. BER analysis for PSK and DPSK}

High-rate communications over wireless links in the sub$\mathrm{THz}$ domain demand tremendous amount of power. For this reason, constant envelope modulations are valuable since they offer an efficient use of amplifiers. Yet, coherent Phase Shift Keying (PSK) are very sensitive to phase related impairments. Differential PSK (DPSK) has been introduced to enable noncoherent demodulation. It is hence not subject to the cumulative nature of PN. This robustness is achieved at the expense of a noise enhancement $-\simeq 3 \mathrm{~dB}-$ and so a BER degradation. Still, the stronger the phase impairments, the more interesting it is to select a differential modulation. This leaves an open question: With regard to normalized corner frequency, when does DPSK become more robust than PSK? The closed form expressions of PSK and DPSK BER subject to $M_{W}$ PN have been derived in [16]. Fig. 7 depicts the BER performance for a 4-PSK and a 4-DPSK with a Signal-to-Noise Ratio (SNR) of $20 \mathrm{~dB}$ and a frame length of $N=7$ symbols. The aforementioned intuition is confirmed: Tough the DPSK presents a loss in BER performance, it is more robust than the PSK for strong correlated PN. It may be observed on Fig. 7 that the PSK and DPSK performance are equal in a critical value $\gamma_{c}$ independent from the white PN variance. It can also be shown that $\gamma_{c}$ is independent from the SNR. For this reason, $\gamma_{c}$ provides a general criterion for link adaptation. Moreover, it is worth noting that the plotted BER satisfies the condition in Eq. (20). With a sequence length $N=7$, Eq. (20) gives that it is not relevant to consider the correlated PN if $f_{c} \cdot T \leq 0.71$. This agrees with the critical value measured in Fig. 7 of $f_{c} \cdot T \simeq 0.853$ greater than 0.71 . 


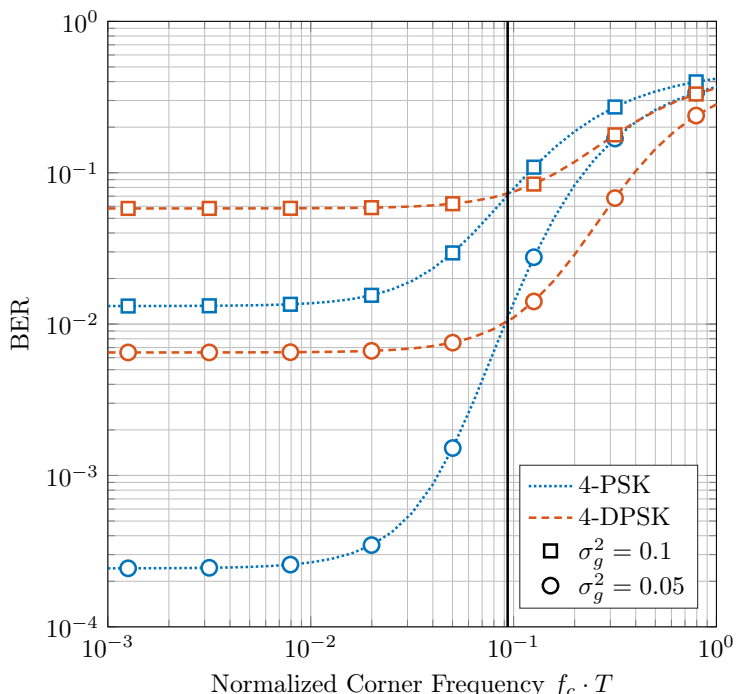

Fig. 7: BER performance of PSK and DPSK for correlated PN.

\section{B. Adaptive systems}

To guarantee robustness or to maximize spectral efficiency, communication systems implement adaptive modulation schemes. The modulation is inferred from a channel estimation. For sub-THz communications, a link adaptation scheme function of the PN level is highly valuable. Indeed, aggregated bands by sub- $\mathrm{THz}$ systems with channel-bonding are wide enough to exhibit differences in oscillators performance between the higher frequencies and the lower ones. In our study, the link adaptation lies in the choice of a differential or coherent modulation and sums up to following question: Is the cumulative PN strong enough to advantage a differential modulation? The link adaptation decision $\delta$ takes the following form

$$
\delta(\boldsymbol{r})=\left\{\begin{array}{ll}
\mathrm{DPSK}, & \text { if } \sigma_{w}^{2} / \sigma_{g}^{2}>\gamma_{c} \\
\mathrm{PSK}, & \text { otherwise }
\end{array},\right.
$$

where $\boldsymbol{r}=\left(r_{1}, \ldots, r_{N}\right)$ is a received sequence of pilots symbols to estimate channel. The link adaptation problem can be expressed as a detection problem

$$
\begin{aligned}
& H_{0}: \sigma_{w}^{2} / \sigma_{g}^{2}>\gamma_{c}, \quad(\text { correlated PN), } \\
& H_{1}: \sigma_{w}^{2} / \sigma_{g}^{2} \leq \gamma_{c}, \quad \text { (uncorrelated PN). }
\end{aligned}
$$

This is a very similar problem to the one studied in Sec. IV-B. The transceiver has to discriminate between $H_{0}$ and $H_{1}$ to select the modulation. We have investigated this detection problem in [16] where a statistic of the received pilot sequence has been proposed to perform the link adaptation.

\section{CONCLUSION}

In this paper, we have addressed the problem of PN modeling for sub-THz communications. We have first introduced two PN models: one correlated, accurate but complex, and another uncorrelated, analytically simpler. Next, we have evaluated the accuracy of the uncorrelated Gaussian PN model with a $\chi^{2}$ goodness of fit test. Simulations have been performed considering realistic PN corresponding to a state-of-the-art $200 \mathrm{GHz}$ oscillator. We have demonstrated that the Gaussian PN model is accurate when the oscillator corner frequency remains small compared to the system bandwidth. This result has been confirmed by deriving the closed-form expression of the LLR between the correlated and uncorrelated PN models. Given the number of samples in a frame and the normalized corner frequency, an analytical condition has been proposed to select the best PN model between correlated and uncorrelated. Simulation results have shown that an uncorrelated Gaussian process is an appropriate PN model for sub- $\mathrm{THz}$ communication systems. Finally, a practical application of model selection has been presented. We have investigated a link adaptation scheme where the transceiver selects the most robust modulation between a coherent and a differential one with regard to the PN performance. Though the results of the paper are presented within the context of sub- $\mathrm{THz}$ communication, the analysis remain true for other scenarios such as satellite communications or next generation of millimeterwave communication $(5 \mathrm{G})$.

\section{REFERENCES}

[1] G. Fettweis, F. Guderian, and S. Krone, "Entering the path towards Terabit/s wireless links," in 2011 Design, Automation Test in Europe, March 2011, pp. 1-6.

[2] M. Voicu, D. Pepe, and D. Zito, "Performance and Trends in MillimetreWave CMOS Oscillators for Emerging Wireless Applications," International Journal of Microwave Science and Technology, vol. 2013, p. 6, 2013.

[3] C. Hager, A. G. i Amat, A. Alvarado, and E. Agrell, "Design of APSK Constellations for Coherent Optical Channels with Nonlinear Phase Noise," IEEE Transactions on Communications, vol. 61, no. 8 pp. 3362-3373, August 2013.

[4] G. J. Foschini, R. D. Gitlin, and S. B. Weinstein, "On the selection of a two-dimensional signal constellation in the presence of phase jitter and gaussian noise," The Bell System Technical Journal, vol. 52, no. 6 , pp. 927-965, July 1973.

[5] M. R. Khanzadi, D. Kuylenstierna, A. Panahi, T. Eriksson, and H. Zirath, "Calculation of the Performance of Communication Systems From Measured Oscillator Phase Noise," IEEE Transactions on Circuits and Systems I: Regular Papers, vol. 61, no. 5, pp. 1553-1565, May 2014.

[6] H. Mehrpouyan, M. R. Khanzadi, M. Matthaiou, A. M. Sayeed, R. Schober, and Y. Hua, "Improving bandwidth efficiency in E-band communication systems," IEEE Communications Magazine, vol. 52, no. 3, pp. 121-128, March 2014.

[7] A. Demir, "Computing Timing Jitter From Phase Noise Spectra for Oscillators and Phase-Locked Loops With White and 1/f Noise," IEEE Transactions on Circuits and Systems I: Regular Papers, vol. 53, no. 9, pp. 1869-1884, Sept 2006.

[8] S. Li, D. Fritsche, C. Carta, and F. Ellinger, “A 200-GHz Sub-Harmonic Injection-Locked Oscillator with $0-\mathrm{dBm}$ Output Power and 3.5DCto-RF-Efficiency," in 2018 IEEE Radio Frequency Integrated Circuits Symposium (RFIC), June 2018, pp. 212-215.

[9] N. J. Kasdin and T. Walter, "Discrete simulation of power law noise [for oscillator stability evaluation]," in Proceedings of the 1992 IEEE Frequency Control Symposium, May 1992, pp. 274-283.

[10] L. Pometcu and R. D'Errico, "Characterization of Sub-THz and mmWave Propagation Channel for Indoor Scenarios," in 12th European Association on Antennas and Propagation (EurAAP 18), Apr 2018.

[11] M. Martalo, C. Tripodi, and R. Raheli, "On the information rate of phase noise-limited communications," in 2013 Information Theory and Applications Workshop (ITA), Feb 2013, pp. 1-7.

[12] R. Krishnan, A. G. i Amat, T. Eriksson, and G. Colavolpe, "Constellation Optimization in the Presence of Strong Phase Noise," IEEE Transactions on Communications, vol. 61, no. 12, pp. 5056-5066, December 2013.

[13] S. Bicaïs, J. Doré, and J. G. Jimenez, "On the Optimum Demodulation in the Presence of Gaussian Phase Noise," in 2018 International Conference on Telecommunications (ICT), June 2018.

[14] T. Moon and W. Stirling, Mathematical Methods and Algorithms for Signal Processing. Prentice Hall, 2000.

[15] J.-B. Doré, Y. Corre, S. Bicais, J. Palicot, E. Faussurier, D. Kténas, and F. Bader, "Above-90GHz Spectrum and Single-Carrier Waveform as Enablers for Efficient Tbit/s Wireless Communications," in 25th International Conference on Telecommunications (ICT'2018), SaintMalo, France, Jun. 2018.

[16] S. Bicaïs, J. Doré, and J. G. Jimenez, "Adaptive PSK Modulation Scheme in the Presence of Phase Noise," in 2018 Signal Processing Advances in Wireless Communications (SPAWC), June 2018. 